\title{
Estimates for fully anisotropic elliptic equations with a zero order term
}

\author{
A. Alberico*- G. di Blasio ${ }^{\dagger}-$ F. Feo ${ }^{\ddagger}$
}

\begin{abstract}
Integral estimates for weak solutions to a class of Dirichlet problems for nonlinear, fully anisotropic, elliptic equations with a zero order term are obtained using symmetrization techniques.
\end{abstract}

\section{Introduction}

We deal with anisotropic Dirichlet problems of the form

$$
\begin{cases}-\operatorname{div}(a(x, u, \nabla u))+g(x, u)=f(x) & \text { in } \Omega \\ u=0 & \text { on } \partial \Omega,\end{cases}
$$

where $\Omega$ is a bounded open subset in $\mathbb{R}^{N}$, with $N \geq 2, a: \Omega \times \mathbb{R} \times \mathbb{R}^{N} \rightarrow \mathbb{R}^{N}$ is a Carathéodory function such that, for a.e. $x \in \Omega$,

(H1) $a(x, \eta, \xi) \cdot \xi \geq \Phi(\xi) \quad$ for $(\eta, \xi) \in \mathbb{R} \times \mathbb{R}^{N}$,

where $\Phi: \mathbb{R}^{N} \rightarrow[0,+\infty[$ is an $N$-dimensional Young function that does not necessarily verify the $\Delta_{2}$-condition (see definition in $\S 2.1$ below).

Moreover, we suppose that $g:(x, s) \in \Omega \times \mathbb{R} \rightarrow \mathbb{R}$ is a Carathéodory function satisfying the following conditions:

(H2) $g$ is a strictly increasing function in $u$ for fixed $x$;

(H3) for every $r \in \mathbb{R}$, there exists $h_{r} \in L^{1}(\Omega)$ such that

$$
|g(x, s)| \leq h_{r}(x) \quad \text { for a.e. } x \in \Omega \text { and } \forall s \in \mathbb{R} \text { with }|s|<r \text {; }
$$

$$
g(x, s) s \geq b(s) s \quad \text { for a.e. } x \in \Omega \text { and } \forall s \in \mathbb{R},
$$

*Istituto per le Applicazioni del Calcolo "M. Picone"(I.A.C.), Sede di Napoli, Consiglio Nazionale delle Ricerche (C.N.R.), Via P. Castellino 111, 80131 Napoli, Italy. E-mail: a.alberico@iac.cnr.it

${ }^{\dagger}$ Dipartimento di Matematica e Fisica, Università della Campania Luigi Vanvitelli, Viale Lincon, 5 - 81100 Caserta, Italy. E-mail: giuseppina.diblasio@unicampania.it

${ }^{\ddagger}$ Dipartimento di Ingegneria, Università degli Studi di Napoli "Pathenope", Centro Direzionale Isola C4 80143 Napoli, Italy. E-mail: filomena.feo@uniparthenope.it

${ }^{0}$ Mathematics Subject Classifications: 35B45, 35J25, 35J60

Key words: Anisotropic Dirichlet problems, A priori estimates, Anisotropic symmetrization, Rearrangements 
where $b: \mathbb{R} \rightarrow \mathbb{R}$ is a continuous and strictly increasing function such that $b(0)=0$. Finally, $f: \Omega \rightarrow \mathbb{R}$ is a nonnegative measurable function with suitable summability (see $\S 2.3$ below).

We stress that the anisotropy of problem (1.1) is governed by a general $N$-dimensional convex function of the gradient not necessarily of polynomial type. In the last three decades, problems related to differential operators whose growth with respect to the partial derivatives of $u$ is governed by different powers, are widely studied (see e.g. [5, 6, 9, 13, 18, 19, 22, 23, 24, 25, 27]). This interest has led to an extensive investigation also for problems governed by fully anisotropic growth conditions (see [1, 2, 3, 4, 16]) and problems related to different type of anisotropy (see e.g. [8, 11, [17).

Our aim is to prove an estimate in rearrangement form for weak solutions to problem (1.1) via symmetrization methods in the spirit of [16]. The symmetrization techniques enable us to transform the anisotropic problem (1.1) into the following isotropic radial problem

$$
\begin{cases}-\operatorname{div}\left(\frac{\Phi}{|\nabla v|^{2}} \nabla v\right)+b(v)=f^{\star}(x) & \text { in } \Omega^{\star} \\ v=0 & \text { on } \partial \Omega^{\star}\end{cases}
$$

defined in the ball $\Omega^{\star}$ centered at the origin and having the same measure as $\Omega$. Here, $\Phi \triangleleft$ denotes the Klimov symmetrization of $\Phi$ and $f^{\star}$ is the symmetric decreasing rearrangement of $f$ (see definition in $\S 2.2$ below). In contrast with the isotropic case (see e.g. [7, 21, 31, 32, 33] and the bibliography starting with them), in problem (1.2) not only the domain and the data are symmetrized but also the ellipticity condition is subject to an appropriate symmetrization.

In [16] it is showed that the symmetric rearrangement of a solution $u$ to the anisotropic problem (1.1) is pointwise dominated by the radial solution to the isotropic problem (1.2) with no zero order term (i.e. with $b \equiv 0$ ). Here, we obtain an estimate preserving the influence of zero order term. Indeed, we prove an integral (and not pointwise) estimate between a solution $u$ to problem (1.1) and the solution $v$ to problem (1.2), i.e.

$$
\int_{\Omega} A(b(u)) d x \leq \int_{\Omega^{\star}} A(b(v)) d x
$$

for every one-dimensional Young function $A$.

The paper is organized as follows. In Section 2, we recall some background on Young functions, rearrangements and Orlicz-Sobolev spaces. In Section 3, we prove the main result and we give some generalization. Finally, in the Appendix we analyze some questions related to the existence and the uniqueness of a solution to problem (1.2).

\section{Preliminaries}

\section{$2.1 \quad$ Young functions}

Let $\Phi: \mathbb{R}^{N} \rightarrow[0,+\infty$ [ be a (finite-valued) $N$-dimensional Young function, namely an even convex function such that

$$
\Phi(0)=0 \quad \text { and } \quad \lim _{|\xi| \rightarrow+\infty} \Phi(\xi)=+\infty .
$$

A standard case considered in literature is when

$$
\Phi(\xi)=\sum_{i=1}^{N} \lambda_{i}\left|\xi_{i}\right|^{p_{i}} \quad \text { for } \xi \in \mathbb{R}^{N},
$$


for some $\lambda_{i}>0$ and $p_{i}>1$, for any $i=1, \ldots, N$. An extension of (2.1) is given by

$$
\Phi(\xi)=\sum_{i=1}^{N} \Upsilon_{i}\left(\xi_{i}\right) \quad \text { for } \xi \in \mathbb{R}^{N}
$$

where $\Upsilon_{i}$, for $i=1, \ldots, N$, are one-dimensional Young functions vanishing only at zero. For instance, we can choose, for $i=1, \ldots, N$,

$$
\Upsilon_{i}(s)=|s|^{p_{i}}(\log (c+|s|))^{\alpha_{i}} \quad \text { for } s \in \mathbb{R},
$$

where either $p_{i}>1$ and $\alpha_{i} \in \mathbb{R}$ or $p_{i}=1$ and $\alpha_{i} \geq 0$, and the constant $c$ is large enough for $\Upsilon_{i}$ to be convex.

If we define the Young conjugate of $\Phi$ the function $\Phi_{\bullet}\left(\xi^{\prime}\right)=\sup \left\{\xi \cdot \xi^{\prime}-\Phi(\xi): \xi \in \mathbb{R}^{N}\right\}$ for $\xi^{\prime} \in$ $\mathbb{R}^{N}$, the Young inequality tells us that

$$
\xi \cdot \xi^{\prime} \leq \Phi(\xi)+\Phi \cdot\left(\xi^{\prime}\right) \quad \text { for } \xi, \xi^{\prime} \in \mathbb{R}^{N},
$$

where "." stands for scalar product in $\mathbb{R}^{N}$. We observe that the function $\Phi$ • enjoys the same properties as $\Phi$ and is a (finite-valued) $N$-dimensional Young function if

$$
\lim _{|\xi| \rightarrow+\infty} \frac{\Phi(\xi)}{|\xi|}=+\infty
$$

The following result (see [30, Theorem 5.1]) says us when the Young inequality (2.4) holds as equality.

Proposition 2.1 If the derivative of $\Phi$ with respect to a direction $\eta$ is linear in $\eta$, then, given any $\xi_{0}$, the $i$-th component of $\eta, \eta_{i}$, verifies $\eta_{i}=\frac{\partial \Phi}{\partial e_{i}}\left(\xi_{0}\right)$ if and only if $\xi_{0} \cdot \eta=\Phi\left(\xi_{0}\right) \Phi \bullet(\eta)$. Here, $\left\{e_{i}\right\}$ stands for a basis in $\mathbb{R}^{N}$.

Finally, we recall a useful property of an $N$-dimensional Young function that we will use in what follows (see [10, Proposition 6.7]).

Proposition 2.2 Let $\Phi \in C^{1}\left(\mathbb{R}^{N}\right)$ be an $N$-dimensional Young function such that (2.5) holds. Then,

$$
\Phi_{\bullet}\left(\Phi_{\xi}(\xi)\right) \leq \Phi_{\xi}(\xi) \cdot \xi \leq \Phi(2 \xi) .
$$

An $N$-dimensional Young function $\Phi$ is said to satisfy the $\Delta_{2}-$ condition near infinity if there exist constants $C>1$ and $K \geq 0$ such that $\Phi(2 \xi) \leq C \Phi(\xi)$ for $|\xi|>K$. For example, one can easily verify that in (2.2) every function $\Upsilon_{i}$ satisfies the $\Delta_{2}$-condition whenever $\Phi(\xi)$ does. Conversely, if one function $\Upsilon_{i}$ does not satisfy the $\Delta_{2}$-condition (as it happens for $\Upsilon_{i}$ given by (2.3)), then $\Phi(\xi)$ does the same. Another example of a function which does not satisfy the $\Delta_{2}$-condition is given by $\Phi(\xi)=e^{\sum_{i=1}^{N}\left|\xi_{i}\right|^{p_{i}}}-1$ with $p_{i} \geq 1$ for any $i=1, \cdots, N$. Indeed,

$$
\lim _{|\xi| \rightarrow+\infty}\left(\sum_{i=1}^{N}\left|2 \xi_{i}\right|^{p_{i}}-\sum_{i=1}^{N}\left|\xi_{i}\right|^{p_{i}}\right)=\lim _{|\xi| \rightarrow+\infty} \sum_{i=1}^{N}\left|\xi_{i}\right|^{p_{i}}\left(2^{p i}-1\right)=+\infty .
$$

\subsection{Symmetrization}

A precise statement of our result requires the use of classical notions of rearrangement of a function (see e.g. [12]) and of suitable symmetrization of a Young function introduced by Klimov in [28]. Let $u$ be a measurable function (continued by 0 outside its domain) fulfilling

$$
\left|\left\{x \in \mathbb{R}^{N}:|u(x)|>t\right\}\right|<+\infty \quad \text { for every } t>0 .
$$


The symmetric decreasing rearrangement of $u$ is the function $u^{\star}: \mathbb{R}^{N} \rightarrow[0,+\infty$ [ satisfying

$$
\left\{x \in \mathbb{R}^{N}: u^{\star}(x)>t\right\}=\left\{x \in \mathbb{R}^{N}:|u(x)|>t\right\}^{\star} \quad \text { for } t>0 .
$$

The decreasing rearrangement $u^{*}$ of $u$ is defined as

$$
u^{*}(s)=\sup \left\{t>0: \mu_{u}(t)>s\right\} \quad \text { for } s \geq 0,
$$

where $\mu_{u}(t)=|\{x \in \Omega:|u(x)|>t\}|$ for $t \geq 0$ denotes the distribution function of $u$. Moreover,

$$
u^{\star}(x)=u^{*}\left(\omega_{N}|x|^{N}\right) \quad \text { for a.e. } x \in \mathbb{R}^{N},
$$

where $\omega_{N}$ is the measure of the $N$-dimensional unit ball. Analogously, we define the symmetric increasing rearrangement $u_{\star}$ of $u$ on replacing " $>$ " by " $<$ " in the definitions of the sets in (2.7) and (2.8).

Let $\Phi$ be an $N$-dimensional Young function. We denote by $\Phi_{\diamond}: \mathbb{R} \rightarrow[0,+\infty[$ the symmetrization of $\Phi$ introduced in [28] (see also [15, 16]). It is the one-dimensional Young function defined by

$$
\Phi(|\xi|)=\Phi_{\bullet \star \bullet}(\xi) \quad \text { for } \xi \in \mathbb{R}^{N},
$$

namely it is the composition of Young conjugation, symmetric increasing rearrangement and Young conjugate again. We stress that the functions $\Phi_{\star}$ and $\Phi_{\star}$ are not equals in general, but they are always equivalent, i.e. there exist two positive constants $K_{1}$ and $K_{2}$ such that

$$
\Phi_{\star}\left(K_{1} \xi\right) \leq \Phi_{\star}(|\xi|) \leq \Phi_{\star}\left(K_{2} \xi\right) \quad \text { for } \xi \in \mathbb{R}^{N} .
$$

Nevertheless, $\Phi_{\star}(|\cdot|)=\Phi_{\star}(\cdot)$ if and only if $\Phi$ is radial, i.e. $\Phi=\Phi_{\star}$.

We emphasize that, if $\Phi$ verifies (2.5), then

$$
\lim _{s \rightarrow+\infty} \frac{\Phi(s)}{s}=+\infty
$$

Moreover, the Young function $\Phi$ verifies the $\triangle_{2}$-condition whenever $\Phi$ does. For the convenience of the reader we give the details. Since $\Phi(\xi) \rightarrow+\infty$ as $|\xi| \rightarrow+\infty$, there exists $M>0$ such that $\{\xi: \Phi(\xi)>t\} \cap\{\xi:|\xi|>M\}=\{\xi: \Phi(\xi)>t\}$ for every $t>0$. The function $\Phi(\xi)$ verifies the $\triangle_{2}$-condition if and only if $\left\{\xi: \Phi(\xi)>\frac{t}{c}\right\} \subseteq\{\xi: \Phi(2 \xi)>t\}$. The assert follows observing the equimisurability of $\Phi$ and $\Phi_{\star}$, the equality $\left\{\xi: \Phi_{\star}(\xi)>\tau\right\}=\mathbb{R}^{N} \backslash B(0, R)$ with $R^{N}=\frac{1}{\omega_{N}}|\{\xi: \Phi(\xi)>\tau\}|$ and the equivalence between $\Phi_{\diamond}$ and $\Phi_{\star}$.

When $\Phi$ is given by (2.1), easy calculations show that

$$
\Phi(|\xi|)=\Lambda|\xi|^{\bar{p}}
$$

where $\bar{p}$ is the harmonic mean of exponents $p_{1}, \ldots, p_{N}$ and $\Lambda$ is a suitable positive constant. In the more general case (2.2), it is possible to show that

$$
\Phi^{-1}(r) \approx\left(\prod_{i=1}^{N} \Upsilon_{i}^{-1}(\cdot)\right)^{\frac{1}{N}}(r) \quad \text { for } r \geq 0,
$$

where $\Upsilon_{i}^{-1}$ denotes the inverse function of $\Upsilon_{i}$ in $[0,+\infty)$. From (2.11), we deduce that

$$
\Phi(s) \approx|s|^{\bar{p}}(\log (c+|s|))^{\frac{\bar{p}}{N} \sum_{i=1}^{N} \frac{\alpha_{i}}{p_{i}}}
$$

near infinity when $\Upsilon_{i}$ is given by (2.3) . 
Now, let us define the following function

$$
\Psi_{\triangleleft}(s)= \begin{cases}\frac{\Phi(s)}{s} & \text { for } s>0 \\ 0 & \text { for } s=0 .\end{cases}
$$

It is not-decreasing and, if, in addiction,

$$
\lim _{s \rightarrow 0^{+}} \frac{\Phi(s)}{s}=0
$$

then it is strictly increasing in $\left[s_{0},+\infty\right)$ with

$$
s_{0}=\sup \{s \geq 0: \Phi \triangleleft(s)=0\} .
$$

Moreover,

$$
\Phi_{\diamond}(r) \leq \Phi_{\diamond}\left(\Psi_{\bullet}^{-1}(r)\right) \quad \text { for } \quad r \geq 0,
$$

where $\Psi^{-1}$ denotes the inverse of $\Psi$ restricted to $\left[s_{0},+\infty\right)$.

We recall that in the anisotropic setting a Polya-Szegö principle holds (see [16]). More precisely, let $u$ be a weakly differentiable function in $\mathbb{R}^{N}$ satisfying (2.7) and such that $\int_{\mathbb{R}^{N}} \Phi(\nabla u) d x<+\infty$. Then, $u^{\star}$ is weakly differentiable in $\mathbb{R}^{N}$ and

$$
\int_{\mathbb{R}^{N}} \Phi\left(\left|\nabla u^{\star}\right|\right) d x \leq \int_{\mathbb{R}^{N}} \Phi(\nabla u) d x
$$

\subsection{Weak solutions and Orlicz Spaces}

In according to the ellipticity condition (H1), we look for weak solution $u$ to problem (1.1) in the class $V_{0}^{1, \Phi}(\Omega)$ of real-valued functions $u$ in $\Omega$, whose continuation by 0 outside $\Omega$ is weakly differentiable in $\mathbb{R}^{N}$ and satisfies $\int_{\Omega} \Phi(\nabla u) d x<\infty$. Let us suppose that the datum $f$ is such that

$$
\int_{\Omega} \Phi \cdot(f(x)) d x<+\infty .
$$

A function $u \in V_{0}^{1, \Phi}(\Omega)$ is a weak solution to problem (1.1) if $b(u) \in L^{1}(\Omega), u b(u) \in L^{1}(\Omega)$ and

$$
\int_{\Omega} a(x, u, \nabla u) \cdot \nabla \varphi d x+\int_{\Omega} g(x, u) \varphi d x=\int_{\Omega} f \varphi d x
$$

for every $\varphi \in V_{0}^{1, \Phi}(\Omega) \cap L^{\infty}(\Omega)$ and for $\varphi=u$.

We recall that $V_{0}^{1, \Phi}(\Omega)$ is always a convex set, but not necessary a linear space since no $\Delta_{2}$-condition on $\Phi$ is required.

Let $\Omega$ be a measurable subset in $\mathbb{R}^{N}$ having finite Lebesgue measure, we say that $A:[0, \infty) \rightarrow$ $[0, \infty)$ is a one-dimensional Young function if it is a convex, left-continuous function, vanishing at 0 , and neither identically equal to 0 , nor to $+\infty$. The Orlicz space $L_{A}(\Omega)$, associated with the Young function $A$, is the set of all measurable functions $g$ in $\Omega$ for which the Luxemburg norm

$$
\|g\|_{L_{A}(\Omega)}=\inf \left\{\lambda>0: \int_{\Omega} A\left(\frac{|g(x)|}{\lambda}\right) d x \leq 1\right\}
$$

is finite. The functional $\|\cdot\|_{L_{A}(\Omega)}$ is a norm on $L_{A}(\Omega)$ which renders the latter a Banach function space. A generalized Hölder inequality

$$
\int_{\Omega}|f g| d x \leq 2\|f\|_{L_{A}(\Omega)}\|g\|_{L_{A_{\bullet}}(\Omega)}
$$


holds for every $f \in L_{A}(\Omega)$ and $g \in L_{A_{\bullet}}(\Omega)$, where $A_{\bullet}$ is the Young conjugate of $A$.

We define the space $E_{A}(\Omega)$ as the closure of $L^{\infty}(\Omega)$ in $L_{A}(\Omega)$. In general $E_{A}(\Omega) \subset L_{A}(\Omega)$ unless $A$ satisfies the $\Delta_{2}$-condition. The space $L_{A}(\Omega)$ is the dual space of $E_{A \bullet}(\Omega)$ and the duality pairing is given by

$$
<f, g>=\int_{\Omega} f g d x
$$

for $f \in L_{A}(\Omega)$ and $g \in E_{A \bullet}(\Omega)$.

The Orlicz-Sobolev space $W^{1} L_{A}(\Omega)$ is defined requiring that $u$ and $|\nabla u|$ are in $L_{A}(\Omega)$. It is a Banach function space equipped with the norm $\|u\|_{W^{1} L_{A}(\Omega)}=\|\mid \nabla u\|_{L_{A}(\Omega)}$.

Finally, we define $W_{0}^{1} L_{A}(\Omega)$ as the closure of $\mathcal{D}(\Omega)$ in $W^{1} L_{A}(\Omega)$ with respect to the weak topology $\sigma\left(L_{A}(\Omega), E_{A \bullet}(\Omega)\right)$. For more details about Orlicz spaces, we refer to [29].

Now we recall the embedding theorem for anisotropic Orlicz-Sobolev spaces (see [15]). Assume that $\Phi$ is an $N$-dimensional Young function fulfilling

$$
\int_{0}\left(\frac{s}{\Phi(s)}\right)^{\frac{1}{N-1}} d s<\infty
$$

and let $\Phi_{N}:[0, \infty) \rightarrow[0, \infty]$ be the Sobolev conjugate of $\Phi$ defined as

$$
\Phi_{N}(s)=\Phi_{\triangleleft}\left(H_{\Phi}^{-1}(s)\right) \quad \text { for } s \geq 0 \quad \text { where } \quad H_{\Phi}(r)=\left(\int_{0}^{r}\left(\frac{s}{\Phi(s)}\right)^{\frac{1}{N-1}} d s\right)^{\frac{1}{N^{\prime}}} \quad \text { for } r \geq 0 \text {. }
$$

If

$$
\int^{+\infty}\left(\frac{s}{\Phi(s)}\right)^{\frac{1}{N-1}} d s<\infty
$$

then any function $u \in V_{0}^{1, \Phi}(\Omega)$ is essentially bounded.

If, instead

$$
\int^{+\infty}\left(\frac{s}{\Phi(s)}\right)^{\frac{1}{N-1}} d s=\infty
$$

then

$$
\int_{\Omega} \Phi_{N}(c u(x)) d x<\infty
$$

for any function $u \in V_{0}^{1, \Phi}(\Omega)$ and for every $c \in \mathbb{R}$.

We stress that we are not interested in the existence of solution to problem (1.1). Nevertheless, thanks to the embedding theorem for anisotropic Orlicz-Sobolev spaces above, we give some conditions on $a(x, \eta, \xi)$ and $f(x)$ in order to guarantee that (2.18) is well posed. More precisely, assume that condition (2.19) holds. Then, the left-hand side of (2.18) is always finite and the right-hand side of (2.18) is finite if $f \in L^{1}(\Omega)$. Otherwise, if condition (2.20) holds, we shall require some conditions on functions $a$ and $f$. More precisely,

$$
\left\|s^{\frac{1}{N}} f^{* *}(s)\right\|_{L_{\Phi_{\bullet}}(0,|\Omega|)}<\infty
$$

and

$$
\Phi \bullet(a(x, \eta, \xi)) \leq c[\theta(x)+M(\eta)+\Phi(\xi)] \quad \text { for a.e. } x \in \Omega \text { and for }(\eta, \xi) \in \mathbb{R} \times \mathbb{R}^{N},
$$

where $f^{* *}(s)=\frac{1}{s} \int_{0}^{s} f^{*}(r) d r, c$ is a positive constant, $\theta$ is a positive function in $L^{1}(\Omega)$ and $M: \mathbb{R} \rightarrow\left[0,+\infty\left[\right.\right.$ is a continuous function such that $M(\eta) \leq \Phi_{N}(k \eta)$ for some $k>0$ and for every $\eta \in \mathbb{R}$. Note that condition (2.21) is weaker than (2.17). 


\section{Mass comparison results}

\subsection{Symmetrized problem}

Our aim is to prove a comparison between the concentration of a solution $u$ to problem (1.1) and the solution $v$ to a suitable simpler isotropic problem. More precisely, in our case the so-called "symmetrized" problem is

$$
\begin{cases}-\operatorname{div}\left(\frac{\Phi}{|\nabla v|^{2}} \nabla v\right)+b(v)=\tilde{f}(x) & \text { in } \Omega^{\star} \\ v=0 & \text { on } \partial \Omega^{\star}\end{cases}
$$

where

$\left(H^{\star} 1\right) \Phi_{\diamond}(t)$ is a one-dimensional Young function strictly increasing in $[0,+\infty)$ such that (2.10) and (2.13) hold,

$\left(\mathrm{H}^{\star} 2\right) \quad b$ is a continuous strictly increasing function such that $b(0)=0$,

$\left(H^{\star} 3\right) \Omega^{\star}$ is the ball centered at the origin having the same measure as $\Omega$,

$\left(\mathrm{H}^{\star} 4\right) \tilde{f}: \Omega^{\star} \rightarrow \mathbb{R}$ is a nonnegative radially symmetric and decreasing along the radii,

$\left(H^{\star} 5\right) \tilde{f} \in E_{\Theta} \cdot\left(\Omega^{\star}\right)$, where $\Theta: \mathbb{R} \rightarrow[0, \infty)$ is the Young function defined by

$$
\Theta(r)=\int_{0}^{|r|} \frac{\Phi(s)}{s} d s \quad \text { for } r \in \mathbb{R} .
$$

By Proposition 13 of [26], under the previous assumptions, there exists a unique positive weak solution $v \in W_{0}^{1} L_{\Theta}\left(\Omega^{\star}\right) \bigcap\left\{v \in W_{0}^{1} L_{\Theta}\left(\Omega^{\star}\right):\left|\frac{\Phi \star}{(|\nabla v|)}\right| \nabla v \mid \in L_{\Theta}\left(\Omega^{\star}\right)\right\}$ to problem (13.1) (see also [21] and the Appendix for more details and remarks), i.e.

$$
\int_{\Omega^{\star}}\left[\frac{\Phi}{|\nabla v|^{2}} \nabla v \cdot \nabla \varphi+b(v) \varphi\right] d x=\int_{\Omega^{\star}} \tilde{f} \varphi d x \quad \text { for every } \varphi \in W_{0}^{1} L_{\Theta}\left(\Omega^{\star}\right) \cap L^{\infty}\left(\Omega^{\star}\right) .
$$

We stress that $\left|\frac{\Phi_{\bullet}(|\nabla v|)}{|\nabla v|^{2}} \nabla v\right|$ does not necessarily belong to the space $L_{\Theta}\left(\Omega^{\star}\right)$ for every $v \in$ $W_{0}^{1} L_{\Theta}\left(\Omega^{\star}\right)$, since $\Theta$ does not necessarily verify a $\Delta_{2}$-condition. Indeed, the function $\Theta$ verifies the $\Delta_{2}$-condition whenever $\Phi$ does 1 .

We look for solutions in the class $V_{0}^{1, \Phi}\left(\Omega^{\star}\right)$. Using the monotonicity of $\Psi \diamond(r)$, defined as in (2.12), we get

$$
\Theta(r) \leq \Phi,(|r|) \quad \text { for } r \in \mathbb{R} .
$$

\footnotetext{
${ }^{1}$ Fixed $t_{1} \in \mathbb{R}$, using the $\Delta_{2}$-condition for $\Phi$, we get, for $|\xi|>2 t_{1}$,

$$
\Theta(2|\xi|)=\int_{0}^{2 t_{1}} \frac{\Phi_{\triangleleft}(s)}{s} d s+\int_{t_{1}}^{|\xi|} \frac{\Phi_{\triangleleft}(2 s)}{s} d s \leq \Theta(|\xi|)+K_{1} \Theta(|\xi|)
$$

for some $K_{1}>0$. Otherwise, since $\Theta$ is $C^{1}$ and strictly increasing, fixed $t_{2} \in \mathbb{R}$, we obtain, for $|\xi|>t_{2}$,

$$
\frac{\Theta(2|\xi|)}{\Theta(|\xi|)}=\frac{\Theta(2|\xi|)-\Theta\left(2 t_{2}\right)}{\Theta(|\xi|)-\Theta\left(t_{2}\right)} \frac{\Theta(2|\xi|)}{\Theta(2|\xi|)-\Theta\left(2 t_{2}\right)} \frac{\Theta(|\xi|)-\Theta\left(t_{2}\right)}{\Theta(|\xi|)} \geq \frac{\Phi_{\bullet}(2 c)}{\Phi_{\bullet}(c)}(1-\epsilon)
$$

for some $\epsilon>0$ and $t_{2}<c<|\xi|$. If $\Delta_{2}$-condition is not fulfilled, then $\frac{\Theta(2|\xi|)}{\Theta(|\xi|)}>K_{2}$, for $|\xi|>t_{2}$ and for every $K_{2} \in \mathbb{R}$.
} 
For this reason we need to require more summability on datum $\tilde{f}$ with to respect to hypothesis $\left(\mathrm{H}^{\star} 5\right)$ in order to assure that the weak solution $v \in W_{0}^{1} L_{\Theta}\left(\Omega^{\star}\right) \bigcap\left\{v \in W_{0}^{1} L_{\Theta}\left(\Omega^{\star}\right):\left|\frac{\Phi_{\star}(|\nabla v|)}{|\nabla v|^{2}} \nabla v\right| \in L_{\Theta \bullet}\left(\Omega^{\star}\right)\right\}$ belongs to the class $V_{0}^{1, \Phi}\left(\Omega^{\star}\right)$. More precisely, if we require the following additional assumptions $\left(\mathrm{H}^{\star} 6\right)$ either $\lim _{r \rightarrow+\infty} \Psi_{\star}(r)=+\infty \quad$ or $\quad \frac{s^{1 / N}}{N \omega_{N}^{1 / N}} \tilde{f}^{* *}(s)<\lim _{r \rightarrow+\infty} \Psi_{\star}(r) \quad$ for every $s>0$, $\left.\left(\mathrm{H}^{\star} 7\right) \int_{0}^{|\Omega|} \Phi \Psi_{\bullet}^{-1}\left(\frac{s^{1 / N}}{N \omega_{N}^{1 / N}} \tilde{f}^{* *}(s)\right)\right) d s \quad$ is finite, then $v \in V_{0}^{1, \Phi \star}\left(\Omega^{\star}\right)$. Indeed, condition $\left(\mathrm{H}^{\star} 6\right)$, monotonicity of $\Psi^{-1}$ and $\left(\mathrm{H}^{\star} 7\right)$ allow to obtain

$$
\begin{aligned}
\int_{\Omega^{\star}} \Phi_{\diamond}(|\nabla v|) d x & =\int_{0}^{\left|\Omega^{\star}\right|} \Phi\left(\Psi^{-1}\left(\frac{\int_{0}^{r}\left[\tilde{f}^{*}(s)-b\left(v^{*}(s)\right)\right] d s}{N \omega_{N}^{1 / N} r^{1 / N^{\prime}}}\right)\right) d r \\
& \leq \int_{0}^{\left|\Omega^{\star}\right|} \Phi\left(\Psi^{-1}\left(\frac{r^{1 / N} \tilde{f}^{* *}(r)}{N \omega_{N}^{1 / N}}\right)\right) d r<+\infty
\end{aligned}
$$

Using (2.15), it is easy to check that condition $\left(\mathrm{H}^{\star} 7\right)$ implies (2.21) with $f=\tilde{f}$ and that $\Phi_{\bullet \bullet}\left(\left|\frac{\Phi \bullet(|\nabla v|)}{|\nabla v|^{2}} \nabla v\right|\right)$ belongs to $L^{1}\left(\Omega^{\star}\right)$, for every $v \in V_{0}^{1, \Phi}\left(\Omega^{\star}\right)$. Moreover, using also (3.3), we have that

$$
\int_{\Omega^{\star}}\left[\frac{\Phi}{|\nabla v|^{2}} \nabla v \cdot \nabla \varphi+b(v) \varphi\right] d x=\int_{\Omega^{\star}} \tilde{f} \varphi d x \quad \text { holds } \quad \text { for } \varphi \in V_{0}^{1, \Phi}\left(\Omega^{\star}\right) \cap L^{\infty}\left(\Omega^{\star}\right) .
$$

Finally, the symmetry of data assures that the solution $v$ of (3.1) is radially symmetric and moreover $v(x)=v^{\star}(x)$ (for more details, see the proof of Theorem 3.1 below).

\subsection{Statements}

Let us consider problems (1.1) and (3.1). In the following theorem, we prove an $L^{\infty}$-norm estimate of the difference between the concentration of solutions in terms of difference between the concentration of data. Moreover, under the additional assumption that datum $f$ is less concentrated than datum $\tilde{f}$, we obtain a comparison between the concentration of a solution $u$ to problem (1.1) and the concentration of the solution $v$ to problem (3.1).

Theorem 3.1 Let $\Phi: \mathbb{R}^{N} \rightarrow[0,+\infty[$ be an $N$-dimensional Young function fulfilling (2.5), such that $\Phi_{\star}(t)$ is strictly increasing in $[0,+\infty)$ verifying (2.13). Assume that $(\mathrm{H} 1)-(\mathrm{H} 4),\left(\mathrm{H}^{\star} 3\right),\left(\mathrm{H}^{\star} 4\right)$, $\left(\mathrm{H}^{\star} 6\right),\left(\mathrm{H}^{\star} 7\right)$ hold and $f$ nonnegative. If $u$ is a (nonnegative) weak solution to (1.1) and $v$ the (nonnegative) weak solution to (3.1), then

$$
\left\|(\mathcal{B}-\widetilde{\mathcal{B}})_{+}\right\|_{L^{\infty}(0,|\Omega|)} \leq\left\|(\mathcal{F}-\widetilde{\mathcal{F}})_{+}\right\|_{L^{\infty}(0,|\Omega|)},
$$

where $(\tau)_{+}:=\max \{0, \tau\}$, and

$$
\begin{array}{ll}
\mathcal{B}(s)=\int_{0}^{s} b\left(u^{*}(t)\right) d t & \widetilde{\mathcal{B}}(s)=\int_{0}^{s} b\left(v^{*}(t)\right) d t \\
\mathcal{F}(s)=\int_{0}^{s} f^{*}(t) d t & \widetilde{\mathcal{F}}(s)=\int_{0}^{s} \widetilde{f}^{*}(t) d t
\end{array}
$$

for $s \in(0,|\Omega|]$. 
An immediate consequence of Theorem 3.1 is the following integral estimate of $b(u)$ in terms of $b(v)$.

Corollary 3.2 Under the same assumptions of Theorem [3.1, if

$$
\mathcal{F}(s) \leq \widetilde{\mathcal{F}}(s) \quad \text { for any } s \in[0,|\Omega|,
$$

then

$$
\mathcal{B}(s) \leq \widetilde{\mathcal{B}}(s) \quad \text { for any } s \in[0,|\Omega|]
$$

Moreover,

$$
\int_{\Omega} A(b(u(x))) d x \leq \int_{\Omega^{\star}} A(b(v(x))) d x
$$

for all convex and non-decreasing function $A:[0,+\infty) \rightarrow[0,+\infty)$ such that $A(0)=0$.

If $b$ is strictly increasing, then

$$
\|u\|_{L^{\infty}(\Omega)} \leq\|v\|_{L^{\infty}\left(\Omega^{\star}\right)} .
$$

Analogous results, for $\Phi$ given by (2.1), are contained in [4, 5].

We note that if $\widetilde{f}=f^{\star}$, then (3.6) holds.

Remark 3.3 The use of Klimov symmetrization gives sharp estimates in previous results. Indeed, in the proof of Theorem 3.1, replacing $\Phi_{\star}$ by $\Phi_{\star}$ and using (2.9), it follows that there exists a constant $K>0$ such that

$$
\left\|(K \mathcal{B}-\widetilde{\mathcal{B}})_{+}\right\|_{L^{\infty}(\Omega)} \leq\left\|(K \mathcal{F}-\widetilde{\mathcal{F}})_{+}\right\|_{L^{\infty}(\Omega)} .
$$

In particular, if there exists $h>0$ such that

$$
\mathcal{F}(s) \leq h \widetilde{\mathcal{F}}(s) \quad \text { for any } s \in[0,|\Omega|],
$$

then

$$
\mathcal{B}(s) \leq h \widetilde{\mathcal{B}}(s) \quad \text { for any } s \in[0,|\Omega|] .
$$

\subsection{Proof of Theorem 3.1}

We define the functions $u_{\kappa, t}: \Omega \rightarrow \mathbb{R}$ as

$$
u_{\kappa, t}(x)= \begin{cases}0 & \text { if }|u(x)| \leq t, \\ (|u(x)|-t) \operatorname{sign}(u(x)) & \text { if } t<|u(x)| \leq t+\kappa \\ \kappa \operatorname{sign}(u(x)) & \text { if } t+\kappa<|u(x)|\end{cases}
$$

for any fixed $t$ and $\kappa>0$. This function can be chosen as test function in (2.18). Using (2.16) and arguing as in [16, we have

$$
\int_{\{t<|u|<t+\kappa\}} \Phi(\nabla u) d x \geq \int_{\{t<u \star<t+\kappa\}} \Phi\left(\left|\nabla u^{\star}\right|\right) d x .
$$

By (H1), we get

$$
\begin{aligned}
\frac{1}{\kappa} \int_{\{t<|u| \leq t+\kappa\}} \Phi(\nabla u) d x & \leq \frac{1}{\kappa} \int_{\{t<|u| \leq t+\kappa\}}(f(x)-g(x, u))(|u(x)|-t) \operatorname{sign}(u(x)) d x \\
& +\int_{\{|u|>t+\kappa\}}(f(x)-g(x, u)) \operatorname{sign}(u(x)) d x .
\end{aligned}
$$


We observe that, since $f$ is nonnegative, standard arguments assure that $u \geq 0$. So, by (H4) and using the monotonicity of $b$, we obtain

$$
\int_{\{|u|>t\}} g(x, u(x)) \operatorname{sign} u d x \geq \int_{\{|u|>t\}} b(u(x)) \operatorname{sign} u d x=\int_{0}^{\mu_{u}(t)} b\left(u^{*}(s)\right) d s .
$$

Letting $\kappa \rightarrow 0^{+}$in (3.9), by (3.8), (3.10) and using the properties of rearrangement, we get

$$
0 \leq-\frac{d}{d t} \int_{\left\{u^{\star}>t\right\}} \Phi \Phi\left(\left|\nabla u^{\star}\right|\right) d x \leq-\frac{d}{d t} \int_{\{|u|>t\}} \Phi(\nabla u) d x \leq \int_{0}^{\mu_{u}(t)}\left(f^{*}(s)-b\left(u^{*}(s)\right)\right) d s .
$$

We claim that

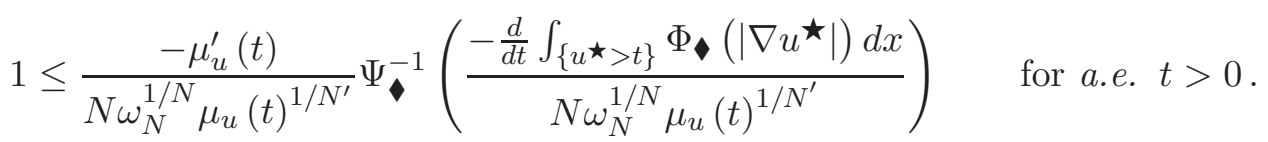

Indeed, since $u$ and $u^{\star}$ are equimeasurable, the Jensen inequality assures that

$$
\Phi\left(\frac{\frac{1}{h} \int_{\{t<u \star<t+h\}}\left|\nabla u^{\star}\right| d x}{\frac{\mu_{u}(t)-\mu_{u}(t+h)}{h}}\right) \leq \frac{\left.\frac{1}{h} \int_{\{t<u}{ }^{\star}<t+h\right\}}{\frac{\mu_{u}(t)-\mu_{u}(t+h)}{h}} \quad \text { for } t \text { and } h>0,
$$

that is

$$
\frac{\frac{1}{h} \int_{\left\{t<u^{\star}<t+h\right\}}\left|\nabla u^{\star}\right| d x}{\frac{\mu_{u}(t)-\mu_{u}(t+h)}{h}} \leq \Psi_{\downarrow}^{-1}\left(\frac{\frac{1}{h} \int_{\left\{t<u^{\star}<t+h\right\}} \Phi_{\star}\left(\left|\nabla u^{\star}\right|\right) d x}{\frac{1}{h} \int_{\left\{t<u^{\star}<t+h\right\}}\left|\nabla u^{\star}\right| d x}\right) \quad \text { for } t \text { and } h>0 .
$$

Finally, coarea formula yields (3.12).

Combining (3.11) and (3.12), we obtain

$$
1 \leq \frac{-\mu_{u}^{\prime}(t)}{N \omega_{N}^{1 / N} \mu_{u}(t)^{1 / N^{\prime}}} \Psi^{-1}\left(\frac{\int_{0}^{\mu_{u}(t)}\left(f^{*}(s)-b\left(u^{*}(s)\right)\right) d s}{N \omega_{N}^{1 / N} \mu_{u}(t)^{1 / N^{\prime}}}\right) \quad \text { for a.e. } t>0 .
$$

By standard arguments, it follows that

$$
-\left(u^{*}(s)\right)^{\prime} \leq \frac{1}{N \omega_{N}^{1 / N} s^{1 / N^{\prime}}} \Psi^{-1}\left(\frac{\mathcal{F}(s)-\mathcal{B}(s)}{N \omega_{N}^{1 / N} s^{1 / N^{\prime}}}\right) \quad \text { for } \quad s \in(0,|\Omega|) .
$$

By (3.4), the derivative of $\mathcal{B}$ equals

$$
\mathcal{B}^{\prime}(s)=b\left(u^{*}(s)\right) \quad \text { for a.e. } s \in(0,|\Omega|) .
$$

Equations (3.4), (3.13) and (3.14) yield

$$
\left\{\begin{array}{l}
\left.N \omega_{N}^{1 / N} s^{1 / N^{\prime}} \Psi\left(N \omega_{N}^{1 / N} s^{1 / N^{\prime}}\left(-\frac{d}{d s}\left(\gamma\left(\mathcal{B}^{\prime}(s)\right)\right)\right)\right)+\mathcal{B}(s) \leq \mathcal{F}(s) \quad \text { for } s \in(0,|\Omega|]\right) \\
\mathcal{B}(0)=0, \quad \mathcal{B}^{\prime}(|\Omega|)=0
\end{array}\right.
$$

where $\gamma(\sigma)=b^{-1}(\sigma)$, the inverse function of $b$.

Now let us consider problem (3.1). The solution $v$ to problem (3.1) is unique and the symmetry of data assures that $v(x)=v(|x|)$, i.e. $v$ is positive and radially symmetric. Moreover, setting $s=$ $\omega_{N}|x|^{N}$ and $\widetilde{v}(s)=v\left(\left(s / \omega_{N}\right)^{1 / N}\right)$, we get, for all $s \in[0,|\Omega|]$,

$$
-\frac{\Phi \downarrow\left(\left|\widetilde{v}^{\prime}(s)\right| N \omega_{N}^{1 / N} s^{1 / N^{\prime}}\right)}{\widetilde{v}^{\prime}(s)}=\int_{0}^{s}\left(\widetilde{f}^{*}(\sigma)-b(\widetilde{v}(\sigma))\right) d \sigma .
$$


Arguing as in (3.11), we have that the integral on the right-hand side of (3.16) is positive and then this fact guarantees that

$$
\frac{\Phi \cdot\left(\left|\widetilde{v}^{\prime}(s)\right| N \omega_{N}^{1 / N} s^{1 / N^{\prime}}\right)}{\widetilde{v}^{\prime}(s)} \leq 0 .
$$

This means that $v(x)=v^{\star}(x)$, since $\Phi$ a positive function.

By the properties of $v$, we can repeat arguments used to prove (3.13) replacing all the inequalities by equalities (see also [20, Lemma 1.32]) and obtaining

$$
-\left(v^{*}(s)\right)^{\prime}=\frac{1}{N \omega_{N}^{1 / N} s^{1 / N^{\prime}}} \Psi^{-1}\left(\frac{\widetilde{\mathcal{F}}(s)-\widetilde{\mathcal{B}}(s)}{N \omega_{N}^{1 / N} s^{1 / N^{\prime}}}\right),
$$

and thus

$$
\left\{\begin{array}{l}
\left.N \omega_{N}^{1 / N} s^{1 / N^{\prime}} \Psi \cdot\left[N \omega_{N}^{1 / N} s^{1 / N^{\prime}}\left(-\frac{d}{d s}\left(\gamma\left(\widetilde{\mathcal{B}}^{\prime}(s)\right)\right)\right)\right]+\widetilde{\mathcal{B}}(s)=\widetilde{\mathcal{F}}(s) \quad \text { for } s \in(0,|\Omega|]\right) \\
\widetilde{\mathcal{B}}(0)=0, \quad \widetilde{\mathcal{B}^{\prime}}(|\Omega|)=0 .
\end{array}\right.
$$

Since $\mathcal{B}, \widetilde{\mathcal{B}} \in \mathcal{C}([0,|\Omega|])$, there exists $\bar{s} \in(0,|\Omega|)$ such that

$$
\left\|(\mathcal{B}-\widetilde{\mathcal{B}})_{+}\right\|_{L^{\infty}(0,|\Omega|)}=(\mathcal{B}-\widetilde{\mathcal{B}})(\bar{s}) .
$$

We argue by contradiction. Suppose that

$$
(\mathcal{B}-\widetilde{\mathcal{B}})(\bar{s})>\left\|(\mathcal{F}-\widetilde{\mathcal{F}})_{+}\right\|_{L^{\infty}(0,|\Omega|)} .
$$

If $\bar{s}<|\Omega|$, combining (3.15) and (3.17) yields

$$
\begin{aligned}
& N \omega_{N}^{1 / N} s^{1 / N^{\prime}}\left[\Psi \cdot\left(N \omega_{N}^{1 / N} s^{1 / N^{\prime}}\left(-\frac{d}{d s}\left(\gamma\left(\mathcal{B}^{\prime}(s)\right)\right)\right)\right)-\Psi\left(N \omega_{N}^{1 / N} s^{1 / N^{\prime}}\left(-\frac{d}{d s}\left(\gamma\left(\widetilde{\mathcal{B}^{\prime}}(s)\right)\right)\right)\right)\right] \\
& \leq \mathcal{F}(s)-\widetilde{\mathcal{F}}(s)+\widetilde{\mathcal{B}}(s)-\mathcal{B}(s) .
\end{aligned}
$$

By (3.18), it follows that

$$
\mathcal{F}(s)-\widetilde{\mathcal{F}}(s)+\widetilde{\mathcal{B}}(s)-\mathcal{B}(s) \leq\left\|(\mathcal{F}-\widetilde{\mathcal{F}})_{+}\right\|_{L^{\infty}(0,|\Omega|)}-(\mathcal{B}-\widetilde{\mathcal{B}})(s)<0 \quad \text { for } s \in(\bar{s}-\varepsilon, \bar{s}+\varepsilon) .
$$

We set

$$
Z=\mathcal{B}-\widetilde{\mathcal{B}} \in W^{2, \infty}(\bar{s}-\varepsilon, \bar{s}+\varepsilon)
$$

Then,

$$
\gamma\left(\mathcal{B}^{\prime}(s)\right)-\gamma\left(\widetilde{\mathcal{B}}^{\prime}(s)\right)=Z^{\prime}(s) c(s)
$$

where

$$
c(s)=\int_{0}^{1} \gamma^{\prime}\left(\tau \mathcal{B}^{\prime}(s)+(1-\tau) \widetilde{\mathcal{B}}^{\prime}(s)\right) d \tau>0 .
$$

As a consequence of (3.19) and (3.20), we obtain

$$
\Psi \diamond\left(N \omega_{N}^{1 / N} s^{1 / N^{\prime}}\left(-\frac{d}{d s}\left(\gamma\left(\mathcal{B}^{\prime}(s)\right)\right)\right)\right)-\Psi \diamond\left(N \omega_{N}^{1 / N} s^{1 / N^{\prime}}\left(-\frac{d}{d s}\left(\gamma\left(\widetilde{\mathcal{B}}^{\prime}(s)\right)\right)\right)\right)<0 .
$$


By the monotonicity of $\Psi^{-1}$ and by (3.22), we get

$$
-\frac{d}{d s}\left(\gamma\left(\mathcal{B}^{\prime}(s)\right)\right)<-\frac{d}{d s}\left(\gamma\left(\widetilde{\mathcal{B}^{\prime}}(s)\right)\right) .
$$

Using (3.21), we can conclude that

$$
-\frac{d}{d s}\left(c(s) Z^{\prime}(s)\right)<0 \quad \text { for } s \in(\bar{s}-\varepsilon, \bar{s}+\varepsilon),
$$

which is in contradiction with the assumption that $Z$ has a maximum in $\bar{s}$.

If $\bar{s}=|\Omega|$, (3.23) holds in $(|\Omega|-\varepsilon,|\Omega|)$ and then $Z^{\prime}(|\Omega|)>0$. But we know that $Z^{\prime}(|\Omega|)=0$.

\subsection{Generalization}

We point out that some generalizations of Theorem 3.1 hold. Let $b_{1}, b_{2}$ be two continuous strictly increasing functions. We say that $b_{1}$ is weaker than $b_{2}$ and we write

$$
b_{1} \prec b_{2},
$$

if they have the same domains and there exists a contraction $2 \rho: \mathbb{R} \rightarrow \mathbb{R}$ such that $b_{1}=\rho \circ b_{2}$.

It is possible to estimate the difference between the concentration of a solution $u \in V_{0}^{1, \Phi}(\Omega)$ to problem (1.1) and the concentration of the solution $v \in V_{0}^{1, \Phi}\left(\Omega^{\star}\right)$ to the following problem

$$
\begin{cases}-\operatorname{div}\left(\frac{\Phi \vee(|\nabla v|)}{|\nabla v|^{2}} \nabla v\right)+\widetilde{b}(v)=\tilde{f}(x) & \text { in } \Omega^{\star} \\ v=0 & \text { on } \partial \Omega^{\star},\end{cases}
$$

under the assumptions $\left(H^{\star} 1\right),\left(H^{\star} 3\right)-\left(H^{\star} 5\right)$ and with $\widetilde{b}$ a continuous strictly increasing function such that $\widetilde{b}(0)=0$ and $\widetilde{b}^{-1} \prec b^{-1}$.

More precisely, we get

$$
\left\|(\mathcal{B}-\widetilde{\mathcal{B}})_{+}\right\|_{L^{\infty}(0,|\Omega|)} \leq\left\|(\mathcal{F}-\widetilde{\mathcal{F}})_{+}\right\|_{L^{\infty}(0,|\Omega|)},
$$

where $\mathcal{F}$ and $\widetilde{\mathcal{F}}$ are defined as in $(3.5)$ and, with abuse of notation, $\mathcal{B}$ and $\widetilde{\mathcal{B}}$ are defined by

$$
\mathcal{B}(s)=\int_{0}^{s} b\left(u^{*}(t)\right) d t \quad \widetilde{\mathcal{B}}(s)=\int_{0}^{s} \widetilde{b}\left(v^{*}(t)\right) d t
$$

for $s \in(0,|\Omega|]$. In particular, if we suppose that

$$
\mathcal{F}(s) \leq \widetilde{\mathcal{F}}(s) \quad \text { for any } s \in[0,|\Omega|],
$$

then

$$
\mathcal{B}(s) \leq \widetilde{\mathcal{B}}(s) \quad \text { for any } s \in[0,|\Omega|]
$$

Finally, we stress that in Theorem 3.1 we can replace assumption that $b$ is a continuous and non decreasing function such that $b(0)=0$ by the following more general condition that $b$ is a maximal monotone graphs in $\mathbb{R}^{2}$ such that $b(0) \ni 0$. Indeed, a maximal monotone graph is a natural generalization of the concept of monotone non-decreasing real function; moreover, the inverse of a maximal monotone graph (that appears in the proof of Theorem 3.1) is again a maximal monotone graph (see [33] for more details). Results in this order of idea are contained in [4] when $\Phi$ is given by (2.1).

\footnotetext{
${ }^{2}$ By contraction, we mean $|\rho(a)-\rho(b)| \leq|a-b|$ for $a, b \in \mathbb{R}$.
} 


\section{Appendix: existence and uniqueness for problem (3.1)}

In this Appendix, we focus our attention on the existence and uniqueness of a solution to the symmetrized problem (3.1).

Let us consider boundary value problems for elliptic equation of the form

$$
\begin{cases}-\operatorname{div}(\mathbf{A}(x, \nabla v))+b(v)=h(x) & \text { in } \Omega \\ v=0 & \text { on } \partial \Omega .\end{cases}
$$

It is well-known that Brézis and Browder in [14] studied the existence for this type of problems when the coefficients of $\mathbf{A}=\left(A_{1}, \ldots, A_{m}\right)$ have polynomial growth and $b$ satisfies a sign condition. Here, we suppose that the principal operator in (4.1) have general growth not necessarily of polynomial type, but governed by a Young function that does not necessarily verify the $\triangle_{2}$-condition. Then, this kind of problems are treated in the framework of the Orlicz-Sobolev spaces and the difficulty comes from the fact that, in general, these spaces are not reflexive.

Questions related to the existence of the solution to such a problem in the form (4.1) have been studied, for example, in [26]. For the convenience of the reader, we recall the following definition and the existence result.

An open subset $\Omega$ of $\mathbb{R}^{N}$ has the segment property if there exist a locally finite open covering $\left\{O_{i}\right\}$ of $\partial \Omega$ and corresponding vectors $\left\{\eta_{i}\right\}$ such that $x+\tau \eta_{i} \in \Omega$ for every $x \in \bar{\Omega} \cap O_{i}$ and $0<\tau<1$, i.e. its boundary is locally the graph of a continuous function.

Theorem 4.1 Let $\Omega$ be a bounded open subset of $\mathbb{R}^{N}$ having the segment property. Let us consider problem (4.1) such that the following conditions hold:

$\left(A_{1}\right)$ Every component $A_{i}$ is a real-valued function defined in $\Omega \times \mathbb{R}^{N}$ which is measurable in $x$ for fixed $\xi$ and continuous in $\xi$ for a.e. fixed $x$.

$\left(A_{2}\right)$ There exist a one-dimensional Young function $M$, a function $\nu \in E_{M_{\bullet}}(\Omega)$ and two constants $c_{1}$ and $c_{2}$ such that

$$
\left|A_{i}(x, \xi)\right| \leq \nu(x)+c_{1} M_{\bullet}^{-1} M\left(c_{2}|\xi|\right)
$$

for a.e. $x \in \Omega$ and all $\xi \in \mathbb{R}^{N}$.

$\left(A_{3}\right)\left(\boldsymbol{A}\left(x, \xi_{1}\right)-\boldsymbol{A}\left(x, \xi_{2}\right)\right) \cdot\left(\xi_{1}-\xi_{2}\right)>0 \quad$ for a.e. $x \in \Omega$ and every $\xi_{1} \neq \xi_{2}$ in $\mathbb{R}^{N}$.

$\left(A_{4}\right)$ There exist two functions $\boldsymbol{b} \in\left(E_{M_{\bullet}}(\Omega)\right)^{N}, b_{0} \in L^{1}(\Omega)$ and two constants $d_{1}$ and $d_{2}$ such that for some fixed element $\varphi \in W_{0}^{1} E_{M}(\Omega) \cap L^{\infty}(\Omega)$

$$
\boldsymbol{A}(x, \xi) \cdot(\xi-\nabla \varphi) \geq d_{1} M\left(d_{2}|\xi|\right)-\boldsymbol{b} \cdot \xi-b_{0}(x)
$$

for a.e. $x \in \Omega$ and all $\xi \in \mathbb{R}^{N}$.

Let $b: \mathbb{R} \rightarrow \mathbb{R}$ be a Carathéodory function such that $b(v) v \geq 0$ for all $v$. Then, given $h \in E_{M \bullet}(\Omega)$, there exists a weak solution $v \in W_{0}^{1} L_{M}(\Omega) \cap\left\{v \in W_{0}^{1} L_{M}(\Omega):|\boldsymbol{A}(x, \nabla v)| \in L_{M}(\Omega)\right\}$ to problem (4.1).

We stress that, when the components of $\mathbf{A}$ have a polynomial growth, i.e. when $M(t)=|t|^{p}$ for $p>1$, then conditions (A1)-(A4) reduce to the classical conditions of the Leray-Lions operators.

The previous existence result runs for $\mathbf{A}(x, \nabla v)=\left(\frac{m(|\nabla v|)}{|\nabla v|} \nabla v\right)$ and $M(t)=\int_{0}^{|t|} m(s) d s$, where $m:[0,+\infty) \rightarrow[0,+\infty)$ is an increasing, right continuous function, $m(t)=0$ if and only if $t=0$ and $m(t) \rightarrow+\infty$ as $t \rightarrow+\infty$. 
Then, it can be applied to the symmetrized problem (3.1) with $\Omega=\Omega^{\star}, m(t)=\frac{\Phi_{\star}(t)}{t}$ and $M(t)=\Theta(t)$, where $\Theta$ is defined as in (3.2). In fact, the assumptions $\left(A_{1}\right)-\left(A_{4}\right)$ are fulfilled with respect to some fixed $\varphi \in W_{0}^{1} E_{\Theta}(\Omega) \cap L^{\infty}(\Omega)$. More precisely, condition $\left(A_{1}\right)$ is obvious since (2.13) holds. Condition $\left(A_{2}\right)$ follows by (3.2) and (2.6) applied to the Young function $\Theta$ :

$$
\Theta(2 t) \geq \int_{t}^{2 t} \frac{\Phi_{\triangleleft}(\tau)}{\tau} d \tau \geq \Phi_{\triangleleft}(t) \geq \Theta \bullet\left(\frac{\Phi_{\triangleleft}(t)}{t}\right) \quad \text { for } t \geq 0,
$$

i.e.

$$
\frac{\Phi(t)}{t} \leq\left(\Theta_{\bullet}\right)^{-1}(\Theta(2 t)) \quad \text { for } t \geq 0
$$

Condition $\left(A_{3}\right)$ is a consequence of the strictly convexity 3 of the function $\xi \in \mathbb{R}^{N} \rightarrow \Theta(|\xi|) \in \mathbb{R}$. Indeed, for all $\xi_{1}, \xi_{2} \in \mathbb{R}^{N}$ with $\xi_{1} \neq \xi_{2}$, we get

$$
\begin{array}{r}
\left(\frac{\Phi\left(\left|\xi_{1}\right|\right)}{\left|\xi_{1}\right|^{2}} \xi_{1}-\frac{\Phi\left(\left|\xi_{2}\right|\right)}{\left|\xi_{2}\right|^{2}} \xi_{2}\right) \cdot\left(\xi_{1}-\xi_{2}\right)=\left(\nabla \Theta\left(\left|\xi_{1}\right|\right)-\nabla \Theta\left(\left|\xi_{2}\right|\right)\right) \cdot\left(\xi_{1}-\xi_{2}\right) \\
>-\left[\Theta\left(\left|\xi_{2}\right|\right)-\Theta\left(\left|\xi_{1}\right|\right)\right]-\left[\Theta\left(\left|\xi_{1}\right|\right)-\Theta\left(\left|\xi_{2}\right|\right)\right]=0 .
\end{array}
$$

By applying Proposition 2.1 $4^{4}$ to the Young function $\Theta$ and the Young inequality (2.4), it follows that

$$
\begin{aligned}
& \left(\frac{\Phi(|\xi|)}{|\xi|^{2}} \xi\right)(\xi-\nabla \varphi(x)) \geq \Theta \bullet\left(\frac{\Phi(|\xi|)}{|\xi|}\right)+\Theta(|\xi|)-\Theta \cdot\left(\frac{\Phi_{\bullet}(|\xi|)}{|\xi|}\right)-\Theta(|\nabla \varphi(x)|) \\
& =\Theta(|\xi|)-\Theta(|\nabla \varphi(x)|),
\end{aligned}
$$

namely condition $\left(A_{4}\right)$ with constants $d_{1}=d_{2}=1, \mathbf{b}(x)=\mathbf{0}$ and $b_{0}(x)=\Theta(|\nabla \varphi(x)|)$ in $L^{1}(\Omega)$.

Finally, we verify the uniqueness of the solution to the symmetrized problem (3.1). It follows in a standard way using the monotonicity of zero order term. Indeed, let $v_{1}$ and $v_{2}$ be two different solutions to (3.1) and let us choose $T_{\delta}\left(\left(v_{1}-v_{2}\right)_{+}\right) / \delta$ as test function in the difference of equations, where $T_{\delta}(t):=\min \{\delta, \max \{t,-\delta\}\}$. Then,

$$
\begin{aligned}
\int_{\Omega^{\star}}\left[\Phi\left(\left|\nabla v_{1}\right|\right) \frac{\nabla v_{1}}{\left|\nabla v_{1}\right|^{2}}-\Phi_{\diamond}\left(\left|\nabla v_{2}\right|\right) \frac{\nabla v_{2}}{\left|\nabla v_{2}\right|^{2}}\right] & \frac{\nabla T_{\delta}\left(\left(v_{1}-v_{2}\right)_{+}\right)}{\delta} d x \\
& +\int_{\Omega^{\star}}\left[b\left(v_{1}\right)-b\left(v_{2}\right)\right] \frac{\nabla T_{\delta}\left(\left(v_{1}-v_{2}\right)_{+}\right)}{\delta} d x=0 .
\end{aligned}
$$

By (A3), (4.2) and the definition of $\left(v_{1}-v_{2}\right)^{+}$, we obtain

$$
\int_{\Omega^{\star}}\left[b\left(v_{1}\right)-b\left(v_{2}\right)\right] \frac{\nabla T_{\delta}\left(\left(v_{1}-v_{2}\right)_{+}\right)}{\delta} d x \leq 0,
$$

and, passing to the limit with $\delta \rightarrow 0$ and using the monotonicity of $b$, it follows $\left|\left\{v_{1}-v_{2}>0\right\}\right|=0$. Replacing $v_{2}$ by $v_{1}$, the assert follows.

Similar arguments show that the solution $v$ to problem (3.1) is nonnegative since $f$ is nonnegative.

\footnotetext{
${ }^{3}$ The function $\Theta(|\xi|)$ verifies the strictly convex condition as $N$-variable function. This follows combining the strictly convexity and the strictly monotonicity (as function of one variable) in $[0,+\infty$ ) of $\Theta$ with the triangular inequality of modulus. We have only to check that $\Theta$ is strictly convex as one variable function. Indeed, the strictly increasing of $\Phi$ implies that $s_{0}=0$ in (2.14) and then $\frac{\Phi_{\bullet}(s)}{s}$ is strictly increasing in $[0,+\infty)$.

${ }^{4}$ For all $\xi, \eta \in \mathbb{R}^{N}$, with $|\eta|=1$, we have that $\frac{\partial}{\partial \eta} \Theta(|\xi|)^{s}=\Theta^{\prime}(|\xi|) \nabla \xi \cdot \eta$ is linear in $\eta$, where $\Theta^{\prime}(t)$ is the derivative of $\Theta(t)$.
} 
Acknowledgements This work has been partially supported by GNAMPA of the Italian INdAM (National Institute of High Mathematics) and "Programma triennale della Ricerca dell'Università degli Studi di Napoli "Parthenope" - Sostegno alla ricerca individuale 2015-2017".

\section{References}

[1] A. Alberico, Boundedness of solutions to anisotropic variational problems, Comm. Part. Diff. Eq. 36 (2011), 470-486, et Erratum et Corrigendum to: "Boundedness of solutions to anisotropic variational problems" [Comm. Part. Diff. Eq. 36 (2011), 470-486], ibidem (2016), DOI: 10.1080/03605302.2015.1132430, 1-3.

[2] A. Alberico, A. Cianchi, Comparison estimates in anisotropic variational problems, Manuscripta Math. 126 (2008), 481-503.

[3] A. Alberico, G. di Blasio, F. Feo, A priori estimates for solutions to anisotropic elliptic problems via symmetrization, Math. Nachr. 290 (2017), 986-1003.

[4] A. Alberico, G. di Blasio, F. Feo, Comparison results for nonlinear anisotropic parabolic problems, Atti Accad. Naz. Lincei Rend. Lincei Mat. Appl. 28 (2017), 305-322.

[5] A. Alberico, G. di Blasio, F. Feo, A symmetrization result for a class of anisotropic elliptic problems, Journal of Mathematical Sciences (United States) 224 (2017), 607-617.

[6] A. Alberico, G. di Blasio, F. Feo, Estimates for solutions to anisotropic elliptic equations with zero order term, Springer Proceedings "Geometric Properties for parabolic and elliptic PDE's"176 (2016), 1-15.

[7] A. Alvino, G. Trombetti, P. L. Lions, On optimization problems with prescribed rearrangements, Nonlinear Anal. 13 (1989), 185-220.

[8] A. Alvino, V. Ferone, G. Trombetti, P. L. Lions, Convex symmetrization and applications, Ann. Inst. H. Poincaré Anal. Non Linéaire 14 (1997), 275-293.

[9] S. Antontsev, M. Chipot, Anisotropic equations: uniqueness and existence results, Diff. Int. Eq. 21 (2008), 401-419.

[10] G. Barletta, A. Cianchi, Dirichlet problems for fully anisotropic elliptic equations, Proc. Roy. Soc. Edinburgh Sect. A 147 (2017), 25-60.

[11] M. Belloni, V. Ferone, B. Kawohl, Isoperimetric inequalities, Wulff shape and related questions for strongly nonlinear elliptic equations, Zeit. Angew. Math. Phys. 54 (2003), 771-789.

[12] C. Bennett, R. Sharpley, Interpolation of operators, Pure and Applied Mathematics, 129, Academic Press, Inc., Boston, MA, 1988.

[13] L. Boccardo, P. Marcellini, C. Sbordone, $L^{\infty}$-regularity for variational problems with sharp nonstandard growth conditions, Boll. Un. Mat. Ital. A 4 (1990), 219-225.

[14] H. Brézis, F. E. Browder, Some properties of higher order Sobolev spaces, J. Math. Pures Appl. IX 61 (1982), 245-259.

[15] A. Cianchi, A fully anisotropic Sobolev inequality, Pacific J. Math. 196 (2000), 283-295.

[16] A. Cianchi, Symmetrization in anisotropic elliptic problems, Comm. Part. Diff. Eq. 32 (2007), 693-717. 
[17] F. Della Pietra, G. di Blasio, Blow-up solutions for some nonlinear elliptic equations involving a Finsler-Laplacian, Publ. Mat. 61 (2017), 213-238.

[18] R. Di Nardo, F. Feo, O. Guibé, Uniqueness result for nonlinear anisotropic elliptic equations. Adv. Differential Equations 18 (2013), 433-458.

[19] R. Di Nardo, F. Feo, Existence and uniqueness for nonlinear anisotropic elliptic equations, Arch. Math. (Basel) 102 (2014), 141-153.

[20] J. I. Díaz, Nonlinear partial differential equations and free boundaries. Vol. I. Elliptic equations, Research Notes in Mathematics, 106, Pitman, Boston, MA, 1985.

[21] J.I. Díaz, Symmetrization of nonlinear elliptic and parabolic problems and applications: A particular overview Progress in partial differential equations: elliptic and parabolic problems (Pont--Mousson, 1991), 116, Pitman Res. Notes Math. Ser., 266, Longman Sci. Tech., Harlow, 1992.

[22] I. Fragalà, F. Gazzola, B. Kawohl, Existence and nonexistence results for anisotropic quasilinear elliptic equations, Ann. Inst. Henri Poincaré, Analyse non linéaire 21 (2004), 715-734.

[23] I. Fragalà, F. Gazzola, G. Lieberman, Regularity and nonexistence results for anisotropic quasilinear elliptic equations in convex domains, Disc. Cont. Dynam. Syst. (2005), 280-286.

[24] N. Fusco, C. Sbordone, Some remarks on the regularity of minima of anisotropic integrals, Comm. Part. Diff. Equat. 18 (1993), 153-167.

[25] M. Giaquinta, Growth conditions and regularity, a counterexample, Manus. Math. 59 (1987), $245-248$.

[26] J. Gossez, V. Mustonen, Variational inequalities in Orlicz-Sobolev spaces, Nonlinear Anal. 11 (1987), 379-392.

[27] P. Marcellini, Regularity of minimizers of integrals of the calculus of variations with non standard growth conditions, Arch. Rat. Mech. Anal. 105 (1989), 267-284.

[28] V. S. Klimov, Isoperimetric inequalities and imbedding theorems, (Russian) Dokl. Akad. Nuak SSSR 217 (1974), 272-275.

[29] M. M. Rao, Z. D. Ren, Applications of Orlicz spaces, Monographs and Textbooks in Pure and Applied Mathematics, 250, Marcel Dekker, Inc., New York, 2002.

[30] M. S. Skaff, Vector valued Orlicz spaces generalized $N$-functions. I., Pacific J. Math. 28 (1969), 193-206.

[31] G. Talenti, Elliptic equations and rearrangements, Ann. Sc. Norm. Sup. Pisa IV 3 (1976), $697-718$.

[32] G. Talenti, Nonlinear elliptic equations, rearrangements of functions and Orlicz spaces, Ann. Mat. Pura Appl. 120 (1979), 160-184.

[33] J. L. Vazquez, Symmetrization and Mass Comparison for Degenerate Nonlinear Parabolic and related Elliptic Equations, Advances in Nonlinear Studies 5 (2005), 87-131. 